Archived version from NCDOCKS Institutional Repository http://libres.uncg.edu/ir/asu/

\title{
Appalachľă
}

$\overline{\text { B O O N E, N O R T H C A R O L I N A }}$

\section{Maternal pre-pregnancy BMI, gestational weight gain, and infant birth weight: A within-family analysis in the United States}

\author{
By: Ji Yan
}

\begin{abstract}
In the United States, the high prevalence of unhealthy preconception body weight and inappropriate gestational weight gain among pregnant women is an important public health concern. However, the relationship among pre-pregnancy BMI, gestational weight gain, and newborn birth weight has not been well established. This study uses a very large dataset of sibling births and a within-family design to thoroughly address this issue. The baseline analysis controlling for mother fixed effects indicates maternal preconception overweight, preconception obesity, and excessive gestational weight gain significantly increase the risk of having a high birth weight baby, respectively, by $1.3,3$ and 3.9 percentage points, while underweight before pregnancy and inadequate gestational weight gain increase the low birth weight incidence by 1.4 and 2 percentage points. The benchmark results are robust in a variety of sensitivity checks. Since poor birth outcomes especially high birth weight and low birth weight have lasting adverse impacts on one's health, education, and socio-economic outcomes later in life, the findings of this research suggest promoting healthy weight among women before pregnancy and preventing inappropriate weight gain during pregnancy can generate significant intergenerational benefits
\end{abstract}

Yan, Ji (2015) "Maternal pre-pregnancy BMI, gestational weight gain, and infant birth weight: A withinfamily analysis in the United States" Economics and Human Biology Volume 18 pp. 1-12 (ISSN 1570-677X) Version of Record Available From (www.sciencedirect.com) 


\title{
Maternal pre-pregnancy BMI, gestational weight gain, and infant birth weight: A within-family analysis in the United States
}

\author{
Ji Yan* \\ Appalachian State University, Department of Economics, 416 Howard Street, Boone, NC 28607, United States
}

\section{Keywords:}

Pre-pregnancy BMI

Gestational weight gain

Birth weight

High birth weight

Low birth weight

\begin{abstract}
A B S T R A C T
In the United States, the high prevalence of unhealthy preconception body weight and inappropriate gestational weight gain among pregnant women is an important public health concern. However, the relationship among pre-pregnancy BMI, gestational weight gain, and newborn birth weight has not been well established. This study uses a very large dataset of sibling births and a within-family design to thoroughly address this issue. The baseline analysis controlling for mother fixed effects indicates maternal preconception overweight, preconception obesity, and excessive gestational weight gain significantly increase the risk of having a high birth weight baby, respectively, by $1.3,3$ and 3.9 percentage points, while underweight before pregnancy and inadequate gestational weight gain increase the low birth weight incidence by 1.4 and 2 percentage points. The benchmark results are robust in a variety of sensitivity checks. Since poor birth outcomes especially high birth weight and low birth weight have lasting adverse impacts on one's health, education, and socio-economic outcomes later in life, the findings of this research suggest promoting healthy weight among women before pregnancy and preventing inappropriate weight gain during pregnancy can generate significant intergenerational benefits.
\end{abstract}

\section{Introduction}

The last two decades have seen a rapid increase in the prevalence of overweight and obesity among American women of reproductive age (National Center for Health Statistics, 2014). Among the women who had delivered live infants, the rate of pre-pregnancy overweight and obesity increased from $23.2 \%$ in $1993-1994$ to $44.8 \%$ in 2009 (Kim et al., 2007; Fisher et al., 2013). As another important concern on pregnant women, excessive gestational weight gain has become increasingly common, even

\footnotetext{
* Tel.: +0018282626123.

E-mail address: yanj@appstate.edu
}

for the women with high body mass index (BMI) before pregnancy (Frederick et al., 2008). In contrast, while maternal obesity is on the rise, pre-pregnancy underweight and gestational inadequate weight gain due to deficient nutrient intake or supplementation remain a significant health problem among low socioeconomic status women. A recent report by the Institute of Medicine (IOM) shows about $32 \%$ of the American low income underweight women had inadequate weight gain during pregnancy in 2007 (IOM, 2009). The high prevalence of unhealthy preconception body weight and inappropriate gestational weight gain among pregnant women is of great concern for the public health community. This is because unhealthy BMI before pregnancy and inappropriate weight gain during pregnancy have been linked to poor birth 
outcomes especially high birth weight (HBW, birth weight $>4000 \mathrm{~g}$ ) and low birth weight (LBW, birth weight $<2500 \mathrm{~g})$.

Both HBW and LBW have lasting negative impacts on one's health, education, and socio-economic outcomes. HBW babies are at a greater risk for overweight, obesity, diabetes, cancer, and other disorders later in life (Hjalgrim et al., 2003; Danielzik et al., 2004; Harder et al., 2007; Wei et al., 2007; Mandl et al., 2009). Moreover, HBW negatively affects cognitive function, learning, and school performance (Richards et al., 2001; Kirkegaard et al., 2006; Cesur and Kelly, 2010). LBW is also a costly birth outcome. The short run excess hospital costs of all the LBW singleton births in 1989 were estimated to be at least 1.07 billion (Almond et al., 2005). In the long term, LBW leads to poor health (Currie and Hyson, 1999), developmental problems (Hack et al., 1995), low educational attainment (Conley and Bennett, 2000), and adverse labor market outcomes (Behrman and Rosenzweig, 2004). In the United States, reducing the incidence of adverse birth outcomes has been an important goal of several large-scale social programs. In this sense, findings from investigating the effects of prepregnancy BMI and gestational weight gain on newborn birth weight will have strong policy implications.

This research is also related to the empirical studies on Barker's fetal origins perspective (Barker, 1990). By Barker's theory and its extension, under-nutrition and over-nutrition during pregnancy due to adverse fetal environment presage some of the most common health disorders in adult life. Recent studies further show the "fetal origins" effects can extend to a broader range of lifetime outcomes and in particular, poor health at birth is a key pathway through which deficient or excessive nutrient intake during fetal development exerts persistent effects (Almond and Currie, 2011). This study will shed new light on such a pathway, since gestational weight gain is a good measure of nutrition in utero and both HBW and LBW are crucial indicators of poor infant health. Furthermore, exploring the impact of pre-pregnancy BMI on birth weight is important too, because the corresponding estimates capture the intergenerational returns by promoting healthy preconception weight among women. Such often-ignored returns should be incorporated into the cost-benefit analysis of various weight management programs for women of reproductive age (Siega-Riz and Laraia, 2006).

To date, the relationship of pre-pregnancy BMI and gestational weight gain to infant birth weight has not been well established. Some studies only look at the association between preconception BMI and birth weight (Abenhaim et al., 2007; Gilboa et al., 2008; McDonald et al., 2010). Lacking gestational weight gain data, these studies are unable to address how pre-pregnancy BMI affects birth weight net of its impact that operates through influencing gestational nutritional intake, an important policy-relevant issue. Their results are quite mixed. The second body of the literature evaluates the effects of gestational weight gain only, without controlling for preconception body weight (Hediger et al., 1989; Abrams and Selvin, 1995; Hickey et al., 1996; Ludwig and Currie, 2010; McDonald et al., 2011). However, the omitted pre-pregnancy BMI clearly biases the parameter estimate on weight gain, since women in various BMI categories differ systematically in gestational weight gain. The third literature investigates the combined effects of the two birth weight inputs (Doherty et al., 2006; Rode et al., 2007; Frederick et al., 2008; Crane et al., 2009; Bodnar et al., 2010). Past studies on this topic, like the previous two strands of research, rarely deal with the unobserved generic, mother, or family level factors. Such factors, when not controlled for, will confound the relationship between the two inputs and birth weight. Indeed, the results of these studies are varying and inconsistent. Finally, the datasets used in the whole literature above are often selective, cross-sectional, and lack of important socioeconomic control variables.

This study uses a very large dataset of about 0.3 million sibling births to thoroughly evaluate the effects of prepregnancy BMI and gestational weight gain on infant birth weight. This dataset has several noteworthy advantages. One, it is constructed from the natality records of all the live births in two states, while much of the prior research uses convenience samples. Two, the dataset records maternal weight and height before pregnancy plus weight at delivery, which makes it feasible to create indicators of both pre-pregnancy BMI and gestational weight gain. Three, it provides a rich set of infant, mother, and family control variables. Four, this panel dataset allows a comparison of birth outcome of singleton births to the same mother. This within-family design can remove the bias due to unobserved mother heterogeneity. Five, the large sample size gives more precise estimates, relative to the previous estimates in the literature.

With this unique dataset, mother fixed effects models are applied to address the effects of maternal preconception body weight and gestational weight gain on the mean birth weight and two tails of the birth weight distribution. The benchmark results suggest preconception overweight, preconception obesity, and excessive gestational weight gain all significantly increase the risk of having a HBW baby, while underweight before pregnancy and inadequate gestational weight gain lead to elevated risks of delivering a LBW infant. Then, a series of sensitivity checks or extensions are performed, where the new results are highly consistent with the baseline estimates.

\section{Data}

The dataset of sibling births is constructed from vital statistics natality records in the states of Pennsylvania and Washington ${ }^{1}$. These natality records in general cover all the live births every year. However, this research focuses on a sample of infants born during 2003-2010 in Pennsylvania and during 2003-2006 in Washington for two reasons. One, it is in 2003 that both states began using the new U.S. Standard Certificates of Live Birth (2003 revision). This revised certificate codes quite a few new

\footnotetext{
1 The public natality birth files are available at the following web of the U.S. Centers for Disease Control and Prevention: www.cdc.gov/nchs/ data_access/Vitalstatsonline.htm. Access to the restricted birth files has been provided by the department of public health in both states.
} 
maternal characteristics important for the present study, such as mother's height, weight at three months before pregnancy, and weight at delivery. Two, the latest data which can be released were collected in 2010 for Pennsylvania and in 2006 for Washington, when this research project was started. Using the records of the universe birth, consecutive sibling births are linked to the same mother over time by mother's name, date of birth, race, and newborn parity. Next, multiple-birth pregnancies (twins, triplets, etc.) are dropped, such that the sample only includes the women with singleton sibling births. In addition, the sample excludes a few women who had more than three births in the short period of newborn sampling or did not reside in either state. The final sample is composed of 148,986 mothers with two live births and 18,991 mothers with three live births, totally 354,945 mother-singleton birth pairs.

Pre-pregnancy BMI is calculated using maternal weight before pregnancy in kilograms $(\mathrm{kg})$ divided by maternal height in meters $(\mathrm{m})$ squared $\left(\mathrm{kg} / \mathrm{m}^{2}\right)$. Then, women are sorted into four categories: $<18.5 \mathrm{~kg} / \mathrm{m}^{2}$ (underweight), $18.5-25 \mathrm{~kg} / \mathrm{m}^{2}$ (normal weight, the reference group), 25$30 \mathrm{~kg} / \mathrm{m}^{2}$ (overweight), and $\geq 30 \mathrm{~kg} / \mathrm{m}^{2}$ (obese). The above cutoff points come from the World Health Organization (WHO)'s criteria for BMI categorization in 1995, which has been commonly adopted (WHO, 1995). Next, a continuous variable of gestational weight gain comes from subtracting maternal pre-pregnancy weight from weight at delivery.

According to the new IOM guideline issued in 2009 (Appendix Table A.1, the upper panel), women in different pre-pregnancy BMI categories should gain appropriate weight within different recommended ranges during pregnancy (IOM, 2009). This is because weight gain within the ranges can provide the optimal tradeoff between lowering the risk of having a LBW baby and reducing the chance of delivering a HBW infant (Rasmussen et al., $2009)^{2}$. The recommended total weight gain ranges are applied to term pregnancies in the data, where gestational weight gain above the ranges is defined as "excessive" and below the ranges as "inadequate". To gauge adequacy of weight gain for preterm pregnancies, we follow the literature to work out the recommended expected weight gain ranges for each BMI group using ranges on weight gain rates in 2nd and 3rd trimester (Bodnar et al., 2010; SiegaRiz et al., 2010; Carreno et al., 2012). Then, we code the women with weight gain above the upper limit of the expected ranges as having "excessive weight gain" and women with weight gain below the lower limit as having "inadequate weight gain".

It is worth noting an old IOM guideline in 1990 (Appendix Table A.1, the lower panel), also widely used in prior research (Frederick et al., 2008; Rode et al., 2007), can be applied to define excessive and inadequate weight gain.

\footnotetext{
${ }^{2}$ Although low gestational weight gain is associated with a lower chance of having an overgrown HBW baby, it can substantially increase the risk of delivering a LBW infant. A similar concern applies to the effects of high gestational weight gain on the incidence of LBW and HBW. As such, all the IOM's recommended pregnancy weight gain ranges are based on a delicate balance of the risks and benefits of varying gestational weight gain on newborn birth weight (Abrams et al., 2000).
}

The 2009 version is generally close to the one in 1990 except for three salient changes (Rasmussen et al., 2010). One, the new guideline uses the 1995 WHO BMI cutoff points to replace the traditional BMI cutoffs from the Metropolitan Life Insurance Table. Two, the 2009 version proposes a closed and conservative total weight gain range for women with term pregnancies who are obese before pregnancy. Three, the 2009 guideline provides ranges of recommended weight gain rates in the 2nd and 3rd trimester. Such modifications have been made because: first, overweight and obesity have become increasingly common in American women before they get pregnant; second, the weight gain recommendation in the old guideline for obese pregnant women has been criticized for being too liberal (DeVader et al., 2007). Whether the 1990 or 2009 guideline is superior has been controversial (Abrams et al., 2000; Thorsdottir et al., 2002; DeVader et al., 2007; Rasmussen et al., 2010). Staying neutral in the debate, this research applies both guidelines and compares the results ${ }^{3}$.

Three birth weight outcomes are explored in the benchmark analysis below: birth weight (BW), HBW, and LBW. Birth weight, a continuous variable, is the primary indicator of an infant's health in most studies of newborn wellbeing. Augmenting birth weight causally increases educational achievement, height, and earnings in adulthood (Behrman and Rosenzweig, 2004; Black et al., 2007). HBW and LBW are two salient poor birth outcomes which have adverse impacts on a number of lifetime outcomes (Almond and Currie, 2011). In addition, a lot of control variables are used in the regression analysis. They include infant characteristics (infant sex, birth order, birth year and month), parental demographics (age, education, race, and ethnicity $)^{4,5}$, maternal socioeconomic status variables (marital status, Medicaid and other types of infant delivery payment, WIC enrollment), maternal health behavior and health status (prenatal care initiation in the first trimester, prenatal smoking, height, pre-pregnancy risk factors).

\footnotetext{
${ }^{3}$ While the 1990 guideline does not provide a closed weight gain range for obese women, we use $11.5 \mathrm{~kg}$ as the upper limit of this range (the same as the upper limit for overweight women), following the literature (Rode et al., 2007). The old guideline does not specify ranges of weight gain rates, either. But the mean weight gain rates for underweight, normal weight and overweight women are falling in the 2009 recommended ranges (Appendix Table A.1, column 2). As such, the following analysis uses the 2009 ranges of weight gain rates to proxy for the unknown ranges in the 1990 guideline.

${ }^{4}$ Maternal race and ethnicity indicators will not be included in the regressions with mother fixed effects, since they are birth invariant. For illustration purpose, Section 4 still reports the descriptive statistics of maternal race and ethnicity.

${ }^{5}$ About $9 \%$ of the fathers in the dataset have missing values on age, race, or education. To handle it, we use the sample means to substitute for missing values and create dummy variables for regression called "father age/race/education missing". Furthermore, whether sibling infants had the same father cannot be identified due to data limitation. The regression model controlling for a variety of father characteristics basically assumes these characteristics sufficiently capture paternal influence on fetal growth. And the effects of these father level variables such as education are assumed to be the same; even some sibling infants had prenatal exposure to different fathers.
} 
This research also examines four other important birth outcomes conditional on gestational age ${ }^{6}$ : birth weight $z$-score for gestational age (BW Z-Score) ${ }^{7}$, birth weight with gestation controlled for (BW conditional on Gestation), large for gestational age (LGA, birth weight above the 10th percentile for the gestational age), and small for gestational age (SGA, birth weight below the 10 th percentile for the gestational age). The corresponding results on the four outcomes will demonstrate how maternal BMI and weight gain affect gestational ageadjusted fetal growth rate, fetal overgrowth and growth restriction. Furthermore, the gestational age used above comes from the obstetric estimate of gestation in the birth files and several studies report this gestation measure is generally very accurate (Wier et al., 2007; Dietz et al., 2014) ${ }^{8}$.

\section{Methods}

To examine the relationship of maternal pre-pregnancy body weight and gestational weight gain to infant birth weight, the following linear model is used in the benchmark analysis:

$\mathrm{Y}_{\mathrm{ij}}=\alpha+\beta \mathrm{BMI}_{\mathrm{ij}}+\gamma \mathrm{GWG}_{\mathrm{ij}}+\delta \mathrm{X}_{\mathrm{ij}}+\mu_{\mathrm{j}}+\varepsilon_{\mathrm{ij}}$

where $\mathrm{Y}_{\mathrm{ij}}$ is a birth weight measure of infant $\mathrm{i}$ born to mother $\mathrm{j}$; $\mathrm{BMI}_{\mathrm{ij}}$ is a vector of three pre-pregnancy BMI categories; $\mathrm{GWG}_{\mathrm{ij}}$ is a vector of two gestational weight gain indicators; and $\mathrm{X}_{\mathrm{ij}}$ is a rich set of infant, mother, and family level control variables (Voigt et al., 2004). Finally, $\mu_{\mathrm{j}}$ represents the fixed effect of mother $\mathrm{j}$, which captures the unobserved generic, mother, family, and fetal environment characteristics common to the sibling births of this mother. Such unobserved confounding factors are correlated with pre-pregnancy BMI, gestational weight gain, as well as infant birth weight. As such, controlling for these factors by mother fixed effects will give unbiased within-family estimates on the effects of BMI $(\beta)$ and gestational weight gain $(\gamma)$. In addition, this specification groups together gestational weight gain across all BMI categories to increase the precision of estimates, while one extension in Section 4 will relax this restriction and explore the heterogeneous effects.

In Eq. (1) that controls for gestational weight gain, $\gamma$ indicates a partial impact of pre-pregnancy BMI, because it

\footnotetext{
${ }^{6}$ It is interesting to look at the effects of pre-pregnancy BMI and weight gain during pregnancy on gestational age especially preterm birth. However, the literature suggests the effects of maternal weight differ by type of preterm birth (McDonald et al., 2010), but the dataset in this study does not categorize preterm birth. Moreover, if some unexpected spontaneous preterm births interrupt normal weight gain process, it can generate a spurious association between low weight gain and a high risk of preterm birth, thus confounding the true relation. Due to these reasons, we focus on birth weight outcomes conditional on gestational age.

7 Birth weights are converted into BW Z-scores for gestational age in this way: subtracting the gestational age-specific birth weight mean from birth weights then divided by the gestational age-specific standard deviation.

${ }^{8}$ Few infants have missing gestational ages (about $0.5 \%$ ) and we exclude them from the sample. The results are almost the same, when the missing values are imputed with the sample mean or last menstrual period.
}

does not include an indirect effect of BMI on newborn birth weight which operates through changing weight gain in pregnancy $^{9}$. Jansson et al. (2008) illustrates this indirect effect and shows how pre-pregnancy BMI and unhealthy dietary patterns jointly alter maternal metabolic hormones, thereby affecting placental nutrient transport, gestational weight gain, and fetal growth. Such an indirect pathway from pre-pregnancy BMI to birth weight cannot be estimated, without data on dietary patterns. Nevertheless, at least from a health policy perspective, this unquantified indirect impact is neither important nor interesting, to the extent that weight gain in pregnancy can be substantially modified by social and nutrition programs. In contrast, the estimates of the vector $\gamma$ are quite policy relevant, since they inform policy makers that aside from conventional intervention on gestational weight gain, promoting healthy weight before pregnancy among women can generate appreciable extra cross-generational returns.

When the dependent variable is binary such as HBW or LBW, Eq. (1) is essentially a linear probability model. One typical concern on this model is that the estimated marginal effects on the outcome probability are constant (non-diminishing), when the continuous independent variables are increased to very large values. This is a minor issue here, since the main variables of interest maternal body weight and weight gain are dummy variables. In addition, near the mean values of those continuous control variables, the estimates of a linear probability model are generally pretty good compared with nonlinear models (Wooldridge, 2002). As to nonlinear models on HBW and LBW, a fixed effects logit model has a unique feature of yielding unbiased estimates on the logodds ratio of the two dichotomous outcomes for different BMI or weight gain categories. But such estimates only allow a "relative" group-wise comparison, since theoretically this nonlinear model cannot give the true marginal effects of preconception BMI and gestational weight gain at the individual level (Wooldridge, 2002). This is an important reason why the benchmark analysis applies a linear probability model on HBW and LBW with mother fixed effects.

With the above pros and cons of nonlinear specifications in mind, consider the following logit specification with mother fixed effects:

$$
\operatorname{Pr}\left(\mathrm{Z}_{\mathrm{ij}}=1\right)=\frac{e^{\eta+\theta \mathrm{BMI}_{\mathrm{ij}}+\lambda \mathrm{GWG}_{\mathrm{ij}}+\varphi \mathrm{X}_{\mathrm{ij}}+\omega_{\mathrm{j}}+v_{\mathrm{ij}}}}{1+e^{\eta+\theta \mathrm{BMI}_{\mathrm{ij}}+\lambda \mathrm{GWG}_{\mathrm{ij}}+\varphi \mathrm{X}_{\mathrm{ij}}+\omega_{\mathrm{j}}+v_{\mathrm{ij}}}},
$$

where $Z_{\mathrm{ij}}$ is either HBW or LBW; the independent variables are the same as Eq. (1); and $\omega_{\mathrm{j}}$ represents a set of mother fixed effects. Conditional maximum likelihood estimation of Eq. (2) provides unbiased estimates of $\theta$ and $\lambda$. The natural exponents of these estimates give the odds ratios of neonatal HBW or LBW of different pre-pregnancy BMI and gestational weight gain groups. For example, suppose $\hat{\theta}_{1}$ is the coefficient estimate of pre-pregnancy obesity from

\footnotetext{
${ }^{9}$ However, $\gamma$ does contain the indirect effects of preconception BMI on infant birth weight which work through BMI-related maternal morbidities in pregnancy not controlled in Eq. (1), such as gestational diabetes and hypertension.
} 
Table 1

Summary statistics by infant birth weight category.

\begin{tabular}{|c|c|c|c|c|c|c|c|c|}
\hline & \multicolumn{2}{|c|}{ (1) All the infants } & \multicolumn{2}{|c|}{ (2) HBW infants } & \multicolumn{2}{|c|}{ (3) Normal BW infants } & \multicolumn{2}{|c|}{ (4) LBW infants } \\
\hline & Mean & SD & Mean & SD & Mean & SD & Mean & SD \\
\hline Birth weight (g) & 3396.41 & $(537.14)$ & 4262.67 & $(239.84)$ & 3358.97 & $(354.02)$ & 2045.37 & $(498.63)$ \\
\hline HBW & 0.11 & $(0.31)$ & 1.00 & $(0.00)$ & 0.00 & $(0.00)$ & 0.00 & $(0.00)$ \\
\hline LBW & 0.05 & $(0.21)$ & 0.00 & $(0.00)$ & 0.00 & $(0.00)$ & 1.00 & $(0.00)$ \\
\hline Pre-pregnancy obesity & 0.21 & $(0.40)$ & 0.27 & $(0.45)$ & 0.20 & $(0.40)$ & 0.21 & $(0.41)$ \\
\hline Pre-pregnancy overweight & 0.24 & $(0.43)$ & 0.28 & $(0.45)$ & 0.24 & $(0.43)$ & 0.21 & $(0.40)$ \\
\hline Pre-pregnancy underweight & 0.04 & $(0.19)$ & 0.01 & $(0.11)$ & 0.04 & $(0.20)$ & 0.07 & $(0.25)$ \\
\hline Excessive weight gain & 0.52 & $(0.50)$ & 0.72 & $(0.45)$ & 0.51 & $(0.50)$ & 0.30 & $(0.46)$ \\
\hline Inadequate weight gain & 0.19 & $(0.39)$ & 0.09 & $(0.29)$ & 0.20 & $(0.40)$ & 0.34 & $(0.47)$ \\
\hline Infant male & 0.51 & $(0.50)$ & 0.64 & $(0.48)$ & 0.50 & $(0.50)$ & 0.47 & $(0.50)$ \\
\hline Infant birth order & 2.12 & $(1.21)$ & 2.32 & (1.41) & 2.10 & (1.17) & 2.05 & $(1.25)$ \\
\hline Mother's age & 27.64 & $(5.57)$ & 28.83 & $(5.35)$ & 27.56 & $(5.55)$ & 26.35 & $(5.83)$ \\
\hline Mother non-Hispanic White & 0.82 & $(0.38)$ & 0.89 & $(0.31)$ & 0.82 & $(0.38)$ & 0.72 & $(0.45)$ \\
\hline Mother non-Hispanic Black & 0.08 & $(0.28)$ & 0.04 & $(0.21)$ & 0.08 & $(0.28)$ & 0.17 & $(0.37)$ \\
\hline Mother Hispanic & 0.04 & $(0.21)$ & 0.03 & $(0.17)$ & 0.04 & $(0.21)$ & 0.06 & $(0.24)$ \\
\hline Mother Asian & 0.03 & $(0.16)$ & 0.01 & $(0.11)$ & 0.03 & $(0.17)$ & 0.03 & $(0.17)$ \\
\hline Mother education $=12$ years & 0.25 & $(0.43)$ & 0.21 & $(0.41)$ & 0.25 & $(0.44)$ & 0.32 & $(0.47)$ \\
\hline Mother education $=13-15$ years & 0.27 & $(0.44)$ & 0.28 & $(0.45)$ & 0.27 & $(0.44)$ & 0.25 & $(0.43)$ \\
\hline Mother education $\geq 16$ years & 0.34 & $(0.47)$ & 0.39 & $(0.49)$ & 0.34 & $(0.47)$ & 0.22 & $(0.42)$ \\
\hline Mother married & 0.70 & $(0.46)$ & 0.80 & $(0.40)$ & 0.70 & $(0.46)$ & 0.54 & $(0.50)$ \\
\hline First prenatal care in trimester 1 & 0.76 & $(0.43)$ & 0.78 & $(0.42)$ & 0.76 & $(0.43)$ & 0.67 & $(0.47)$ \\
\hline Prenatal smoking & 0.14 & $(0.35)$ & 0.06 & $(0.24)$ & 0.15 & $(0.35)$ & 0.28 & $(0.45)$ \\
\hline Mother in WIC & 0.33 & $(0.47)$ & 0.26 & $(0.44)$ & 0.34 & $(0.47)$ & 0.44 & $(0.50)$ \\
\hline Mother in Medicaid & 0.26 & $(0.44)$ & 0.19 & $(0.39)$ & 0.26 & $(0.44)$ & 0.38 & $(0.49)$ \\
\hline Father's age & 30.37 & $(6.00)$ & 31.21 & $(5.81)$ & 30.31 & $(6.00)$ & 29.54 & $(6.27)$ \\
\hline Father non-Hispanic White & 0.76 & $(0.43)$ & 0.84 & $(0.37)$ & 0.76 & $(0.43)$ & 0.63 & $(0.48)$ \\
\hline Father non-Hispanic Black & 0.09 & $(0.29)$ & 0.06 & $(0.23)$ & 0.09 & $(0.29)$ & 0.17 & $(0.37)$ \\
\hline Father Hispanic & 0.11 & $(0.31)$ & 0.08 & $(0.27)$ & 0.11 & $(0.31)$ & 0.16 & $(0.37)$ \\
\hline Father Asian & 0.02 & $(0.15)$ & 0.01 & $(0.10)$ & 0.02 & $(0.15)$ & 0.02 & $(0.15)$ \\
\hline Father education $=12$ years & 0.29 & $(0.45)$ & 0.25 & $(0.43)$ & 0.29 & $(0.45)$ & 0.34 & $(0.48)$ \\
\hline Father education $=13-15$ years & 0.24 & $(0.42)$ & 0.25 & $(0.44)$ & 0.24 & $(0.42)$ & 0.20 & $(0.40)$ \\
\hline Father education $\geq 16$ years & 0.29 & $(0.45)$ & 0.34 & $(0.47)$ & 0.29 & $(0.45)$ & 0.19 & $(0.40)$ \\
\hline $\mathrm{N}$ & 354,945 & & 38,167 & & 300,639 & & 16,139 & \\
\hline
\end{tabular}

Notes: The full sample which is constructed from vital statistics natality birth records consists of all the mothers having delivered two or three live births in the state of Pennsylvania in 2003-2010 and in the state of Washington in 2003-2006.

Eq. (2) with HBW as the outcome, then $e^{\hat{\theta}_{1}}$ gives the following odds ratio of having a HBW baby for obese women relative to normal weight women:

$\mathrm{OR}_{\mathrm{HBW}}=\frac{[\operatorname{Pr}(\mathrm{HBW}=1 \mid \mathrm{OB}=1) / \operatorname{Pr}(\mathrm{HBW}=0 \mid \mathrm{OB}=1)]}{[\operatorname{Pr}(\mathrm{HBW}=1 \mid \mathrm{NW}=1) / \operatorname{Pr}(\mathrm{HBW}=0 \mid \mathrm{NW}=1)]}$

Clearly, this odds ratio makes a relative comparison on the odds of delivering a HBW infant between obese and normal weight women ${ }^{10}$. If $e^{\hat{\theta}_{1}}$ is greater than 1 , then the odds of having a HBW infant is $e^{\hat{\theta}_{1}}$ times greater for obese women, compared to normal weight women. The corresponding estimates of the other BMI or weight gain categories can be interpreted in a similar manner.

\section{Results}

Table 1 reports the summary statistics of the three primary birth weight outcomes, infant characteristics, and

\footnotetext{
${ }^{10}$ In this comparison, both groups of women have appropriate gestational weight gain within the 2009 (or 1990) IOM recommended ranges.
}

the key parental control variables ${ }^{11}$. Column (1) focuses on the full sample of sibling births. It shows $11 \%$ of all the infants are HBW and $5 \%$ of them are LBW. About $21 \%$ of the babies were delivered by obese women with pre-pregnancy BMI above $30 \mathrm{~kg} / \mathrm{m}^{2}, 24 \%$ of them by overweight women, and $4 \%$ by underweight women. As to weight gain during pregnancy, $52 \%$ of the newborns' mothers gained more than the recommended ranges by the 2009 guideline, and $19 \%$ of them gained less than the recommended ranges (similar results with the 1990 guideline). In addition, most of the mothers in the sample are either non-Hispanic White or non-Hispanic Black and having at least 12 years of education.

Columns (2) to (4) stratify the full sample by birth weight category. Compared with the women having normal birth weight infants (column 3), the women who delivered HBW babies were more likely to be overweight or obese prior to conception and have excessive weight gain in pregnancy (column 2), suggesting over-nutrition is associated with fetal overgrown. Moreover, they were older, more educated, and more likely to be married and

\footnotetext{
${ }^{11}$ Additional birth variant control variables not shown for brevity include other infant delivery payment types and indicators of prepregnancy risk factors.
} 
Pre-pregnancy BMI, gestational weight gain, and infant birth weight: baseline results.

\begin{tabular}{|c|c|c|c|c|c|c|}
\hline & \multicolumn{3}{|c|}{ IOM (2009) Guideline } & \multicolumn{3}{|c|}{ IOM (1990) Guideline } \\
\hline & (1) Birth weight & (2) HBW & (3) LBW & (4) Birth weight & (5) HBW & (6) LBW \\
\hline Pre-pregnancy obesity & $80.264(5.305)^{* * * *}$ & $0.030(0.004)^{* * * *}$ & $-0.013(0.002)^{* * *}$ & $82.713(4.923)^{* * *}$ & $0.032(0.003)^{* * *}$ & $-0.011(0.002)^{* * *}$ \\
\hline Pre-pregnancy overweight & $29.960(3.404)^{* * *}$ & $0.013(0.002)^{* * *}$ & $-0.007(0.002)^{* * *}$ & $24.494(3.632)^{* * * *}$ & $0.015(0.003)^{* * * *}$ & $-0.003(0.002)^{*}$ \\
\hline Pre-pregnancy underweight & $-29.762(6.725)^{* * * *}$ & $-0.010(0.004)^{* * * *}$ & $0.014(0.004)^{* * * *}$ & $-42.082(4.367)^{* * * *}$ & $-0.009(0.003)^{* * * *}$ & $0.013(0.002)^{* * *}$ \\
\hline Excessive weight gain & $125.541(2.507)^{* * * *}$ & $0.039(0.002)^{* * *}$ & $-0.026(0.001)^{* * *}$ & $113.865(2.484)^{* * *}$ & $0.039(0.002)^{* * * *}$ & $-0.020(0.001)^{* * *}$ \\
\hline Inadequate weight gain & $-62.113(3.057)^{* * *}$ & $-0.011(0.002)^{* * *}$ & $0.020(0.002)^{* * * *}$ & $-67.818(2.885)^{* * *}$ & $-0.014(0.002)^{* * *}$ & $0.021(0.002)^{* 2 * *}$ \\
\hline Infant control variables? & $\mathrm{Y}$ & $\mathrm{Y}$ & $\mathrm{Y}$ & $\mathrm{Y}$ & $\mathrm{Y}$ & $\mathrm{Y}$ \\
\hline Parental control variables? & $\mathrm{Y}$ & $\mathrm{Y}$ & $\mathrm{Y}$ & $\mathrm{Y}$ & $\mathrm{Y}$ & $\mathrm{Y}$ \\
\hline Mother fixed effects? & $\mathrm{Y}$ & $\mathrm{Y}$ & $\mathrm{Y}$ & $\mathrm{Y}$ & $\mathrm{Y}$ & $\mathrm{Y}$ \\
\hline $\mathrm{N}$ & 354,945 & 354,945 & 354,945 & 354,945 & 354,945 & 354,945 \\
\hline
\end{tabular}

Notes: All the regressions use the full sibling-birth sample which consists of all the mothers having delivered two or three live births in the state of Pennsylvania in 2003-2010 and in the state of Washington in 2003-2006. Furthermore, columns (1) and (4) apply a linear regression model; columns (2), (3), (5) and (6) use a linear probability model for regression analysis. The infant control variables include infant sex, birth order, birth year and month fixed effects. The parental control variables include mother's age, mother's education, marital status, prenatal care initiation in trimester one, prenatal smoking, mother's height, WIC enrollment, delivery payment types, indicators of pre-pregnancy risk factors, father's age, father's race, and father's education. Robust standard errors clustered at the mother's level are reported in parentheses.

* Significant at $10 \%$ level.

** Significant at $5 \%$ level.

*** Significant at $1 \%$ level.

initiate prenatal care in the first trimester, as well as were less likely to enroll in WIC/Medicaid or smoke in pregnancy. By contrast, the mothers with LBW newborns were more likely than the mothers with normal birth weight infants to be underweight before pregnancy and gain inadequate weight during pregnancy (column 4). Nutrient deficiencies before and during pregnancy thus appear strongly correlated with low socioeconomic status, since the women with LBW babies tended to be younger, less educated, unmarried, and enroll in WIC and Medicaid. Furthermore, these women also had a higher propensity to smoke during pregnancy and initiate prenatal care after the first trimester. Finally, relative to the LBW and normal birth weight infants, the HBW infants generally are more likely to be male and have a high birth order.

Table 2 presents the benchmark results on the effects of pre-pregnancy BMI and gestational weight gain, by estimating Eq. (1) with the full sample of sibling births. All the infant and parental confounding variables mentioned above have been adjusted in the regression ${ }^{12}$. The first three columns use the 2009 guideline to define the two inappropriate weight gain categories. Column (1) shows pre-pregnancy overweight and obesity significantly increase newborn birth weight by about $30 \mathrm{~g}$ and $80 \mathrm{~g}$, while underweight reduces birth weight by $30 \mathrm{~g}$. Moreover, excessive weight gain during pregnancy results in a significant birth weight increase of $126 \mathrm{~g}$, but inadequate weight gain leads to a birth weight decrease of $62 \mathrm{~g}$. Columns (2) and (3) demonstrate the women who are overweight before pregnancy, obese before pregnancy, or gain excessive weight during pregnancy are $1.3,3$, or 3.9 percentage points more likely to have a HBW baby, although such women also have a lower chance of delivering a LBW infant. The two columns also show

\footnotetext{
12 Such confounding variables are likely to affect both maternal weight before or during pregnancy and newborn birth weight. Therefore, it is important to control for them in regression. The coefficient estimates of these control variables are suppressed to save room.
}

while the women who are underweight before conception or gain inadequate weight in pregnancy are somewhat less likely to give birth to a HBW baby, they have a significantly higher risk of delivering a LBW newborn by 1.4 or 2 percentage points, respectively ${ }^{13}$. As discussed above, the set of parameter estimates on preconception body weight capture a partial impact of pre-pregnancy BMI on neonatal birth weight which does not operate through changing gestational weight gain. But they are not small, compared with the corresponding coefficient estimates on weight gain in pregnancy.

In general, the benchmark estimates suggest overnutrition before and during pregnancy impairs infant health mainly at the upper end of the birth weight distribution, while the adverse effects of deficient nutrient intake are manifest at the lower end. While the effects of prepregnancy BMI and gestational weight gain on the mean birth weight are modest, the impacts of the two on HBW and LBW are large. Columns (4)-(6) of Table 2 present similar results, when the IOM 1990 recommendation is applied to define excessive and inadequate weight gain. Since both guidelines give very close results, the subsequent tables only report the estimates when the 2009 guideline is used.

Table 3 applies the baseline specification to subsample analysis. The upper panel stratifies the full sample by number of sibling births. The results are quite similar to

\footnotetext{
13 Additional analysis with mother fixed effects models indicates a negative association between preconception obesity and excessive gestational weight gain. It implies obese women can mitigate the total effect of obesity on the HBW risk by limiting their gestational weight gain to an IOM recommended range, which also helps reducing the LBW risk. Table 2 suggests the obese women with appropriate gestational weight gain are about 3 percentage points more likely to have a HBW infant, but this risk doubles for those obese women who do not take action to limit their gestational weight gain. About $50 \%$ of the normal weight women in the dataset gained excessive weight during pregnancy. Table 2 shows for the women with healthy weight before pregnancy, excessive weight gestational gain still substantially increases the risk of having a HBW baby by 3.9 percentage points. Thus, promoting healthy weight gain during pregnancy is meaningful even for normal weight women.
} 
Table 3

Pre-pregnancy BMI, gestational weight gain, and infant birth weight: by number of births and by state.

\begin{tabular}{|c|c|c|c|c|c|c|}
\hline & \multicolumn{3}{|c|}{ Mothers with two births } & \multicolumn{3}{|c|}{ Mothers with three births } \\
\hline & (1) Birth weight & (2) HBW & (3) LBW & (4) Birth weight & (5) HBW & (6) LBW \\
\hline Pre-pregnancy obesity & $76.433(5.964)^{* * * *}$ & $0.029(0.004)^{* * * *}$ & $-0.014(0.003)^{* * * *}$ & $76.517(11.609)^{* * * *}$ & $0.035(0.008)^{* * * *}$ & $-0.009(0.005)^{*}$ \\
\hline Pre-pregnancy overweight & $25.387(3.823)^{* * *}$ & $0.013(0.003)^{* * * *}$ & $-0.007(0.002)^{* * * *}$ & $37.581(7.487)^{* * * a *}$ & $0.011(0.005)^{* *}$ & $-0.006(0.003)^{*}$ \\
\hline Pre-pregnancy underweight & $-28.330(7.470)^{* * *}$ & $-0.009(0.004)^{* *}$ & $0.013(0.005)^{* * * *}$ & $-36.818(15.497)^{* *}$ & $-0.015(0.008)^{*}$ & $0.015(0.009)^{*}$ \\
\hline Excessive weight gain & $126.166(2.806)^{* * * *}$ & $0.038(0.002)^{* * * *}$ & $-0.027(0.001)^{* * *}$ & $130.414(5.594)^{* * * *}$ & $0.049(0.004)^{*+* 4}$ & $-0.022(0.003)^{*+*+}$ \\
\hline Inadequate weight gain & $-63.111(3.451)^{* * * *}$ & $-0.012(0.002)^{* * *}$ & $0.021(0.002)^{* * * *}$ & $-60.606(6.603)^{* * *}$ & $-0.009(0.004)^{* *}$ & $0.020(0.004)^{* * * *}$ \\
\hline Infant control variables? & $\mathrm{Y}$ & Y & $\mathrm{Y}$ & $\mathrm{Y}$ & $\mathrm{Y}$ & Y \\
\hline Parental control variables? & $\mathrm{Y}$ & $\mathrm{Y}$ & $\mathrm{Y}$ & $\mathrm{Y}$ & $\mathrm{Y}$ & $\mathrm{Y}$ \\
\hline Mother fixed effects? & $\mathrm{Y}$ & $\mathrm{Y}$ & $\mathrm{Y}$ & $\mathrm{Y}$ & $\mathrm{Y}$ & $\mathrm{Y}$ \\
\hline \multirow[t]{3}{*}{$N$} & 297,972 & 297,972 & 297,972 & 56,973 & 56,973 & 56,973 \\
\hline & \multicolumn{3}{|l|}{ Mothers in PA } & \multicolumn{3}{|l|}{ Mothers in WA } \\
\hline & (1) Birth weight & (2) HBW & (3) LBW & (4) Birth weight & (5) HBW & (6) LBW \\
\hline Pre-pregnancy obesity & $71.077(5.680)^{* * * *}$ & $0.031(0.004)^{* * *}$ & $-0.011(0.003)^{* * * *}$ & $92.642(14.224)^{* * * *}$ & $0.027(0.010)^{* * *}$ & $-0.018(0.006)^{*+*+4}$ \\
\hline Pre-pregnancy overweight & $26.978(3.680)^{*+* *}$ & $0.013(0.003)^{* * * *}$ & $-0.007(0.002)^{* * * *}$ & $25.092(8.736)^{*+3 *}$ & $0.012(0.006)^{* *}$ & $-0.006(0.004)^{*}$ \\
\hline Pre-pregnancy underweight & $-30.113(7.186)^{* . * *}$ & $-0.008(0.004)^{* * *}$ & $0.014(0.004)^{*+* *}$ & $-31.166(18.295)^{*}$ & $-0.018(0.011)^{*}$ & $0.017(0.010)^{*}$ \\
\hline Excessive weight gain & $127.650(2.703)^{* * * *}$ & $0.038(0.002)^{* * *}$ & $-0.027(0.001)^{* * *}$ & $121.567(6.566)^{* * *}$ & $0.047(0.005)^{* * * *}$ & $-0.019(0.003)^{* *+*}$ \\
\hline Inadequate weight gain & $-60.858(3.298)^{* * * *}$ & $-0.010(0.002)^{* * *}$ & $0.021(0.002)^{* * * *}$ & $-68.320(7.886)^{* * *}$ & $-0.015(0.005)^{* * *}$ & $0.017(0.004)^{* * * 4}$ \\
\hline Infant control variables? & Y & $\mathrm{Y}$ & $\mathrm{Y}$ & $\mathrm{Y}$ & $\mathrm{Y}$ & $\mathrm{Y}$ \\
\hline Parental control variables? & $\mathrm{Y}$ & $\mathrm{Y}$ & $\mathrm{Y}$ & $\mathrm{Y}$ & Y & $\mathrm{Y}$ \\
\hline Mother fixed effects? & $\mathrm{Y}$ & $\mathrm{Y}$ & $\mathrm{Y}$ & $\mathrm{Y}$ & $\mathrm{Y}$ & $\mathrm{Y}$ \\
\hline$N$ & 304,025 & 304,025 & 304,025 & 50,920 & 50,920 & 50,920 \\
\hline
\end{tabular}

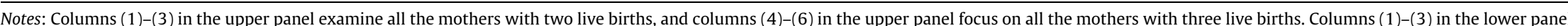

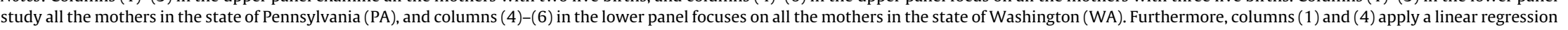

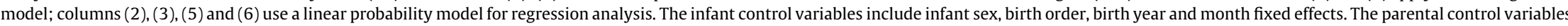

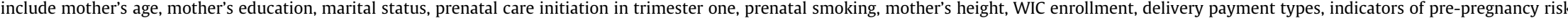
factors, father's age, father's race, and father's education. Robust standard errors clustered at the mother's level are reported in parentheses.

* Significant at $10 \%$ level.

** Significant at $5 \%$ level.

*** Significant at $1 \%$ level. 
Table 2. For the two-birth mothers, preconception overweight, preconception obesity, and excessive weight gain in pregnancy increase the risk of delivering a HBW baby by $1.3,2.9$ and 3.8 percentage points, respectively. In contrast, pre-pregnancy underweight and inadequate gestational weight gain lead to a significant increase in the LBW incidence by 1.3 and 2.1 percentage points (columns 1-3). Similar results hold for the three-birth mothers, in which the estimated impacts of obesity and excessive weight gain on HBW are somewhat larger (columns 4-6). The lower panel considers the Pennsylvania and Washington state subsamples. Again, the findings of either state are consistent with the results from the pooled sample in Table 2.

The left panel of Table 4 examines four other birth outcomes conditional on gestational age. Column (1) shows over-nutrition or under-nutrition before and during pregnancy moderately affects the standardized BW Z-Score for gestational age. Column (2) controls for gestational age. The results, when compared with the total effects on birth weight without controlling for gestational age in Table 2 (column 1), suggest about 60 to $80 \%$ of the total effects come from the impact of the two inputs on birth weight conditional on gestation, with the remainder from affecting gestational age. The estimates in columns (3) and (4) suggest preconception overweight, preconception obesity, and excessive gestational weight gain increase the risk of LGA by 13 to $28 \%$; meanwhile, pre-pregnancy underweight and inadequate weight gain in pregnancy increase the SGA incidence by 16 to $22 \%$.

The right panel restricts the sample to term sibling births (gestational age $>36$ weeks), where the corresponding estimates exclude the effects of the two inputs on birth outcomes which operate through preterm birth. This restriction does not significantly change the sample means of birth weight and HBW, but substantially lower the mean of LBW to $2 \%$. The results on birth weight (column 5 ) are generally consistent with Table 2, except that BMI and weight gain have smaller effects since their impacts on prematurity have been removed. The findings on HBW (column 6) are close to the benchmark results in Table 2, since dropping preterm births has little effect on the upper end of the birth weight distribution and less than $1 \%$ of the preterm babies in the sample are HBW. Finally, since unhealthy body weight and weight gain can increase the LBW incidence through prematurity, restriction to term pregnancies reduces the absolute magnitude of the estimated impacts of the two inputs (column 7). Unlike the estimated effects on HBW, such estimated impacts on LBW in percentage points are not directly comparable with their counterparts in Table 2, due to very different sample means on LBW. Comparing the estimates relative to the respective sample means suggests the effect of weight gain on LBW is still sizable net of its impact on prematurity ${ }^{14}$.

\footnotetext{
${ }^{14}$ For instance, inadequate weight gain increases the LBW incidence among all the infants (both preterm and term pregnancies) by $40 \%$ of the mean: $0.02 / 0.05=0.4$, with the estimate in column (3) of Table 2. Conditional on term pregnancies, inadequate weight gain still increases the LBW incidence by as much as 30\%: $0.006 / 0.02=0.3$, with the estimate in column (7) of Table 4.
}

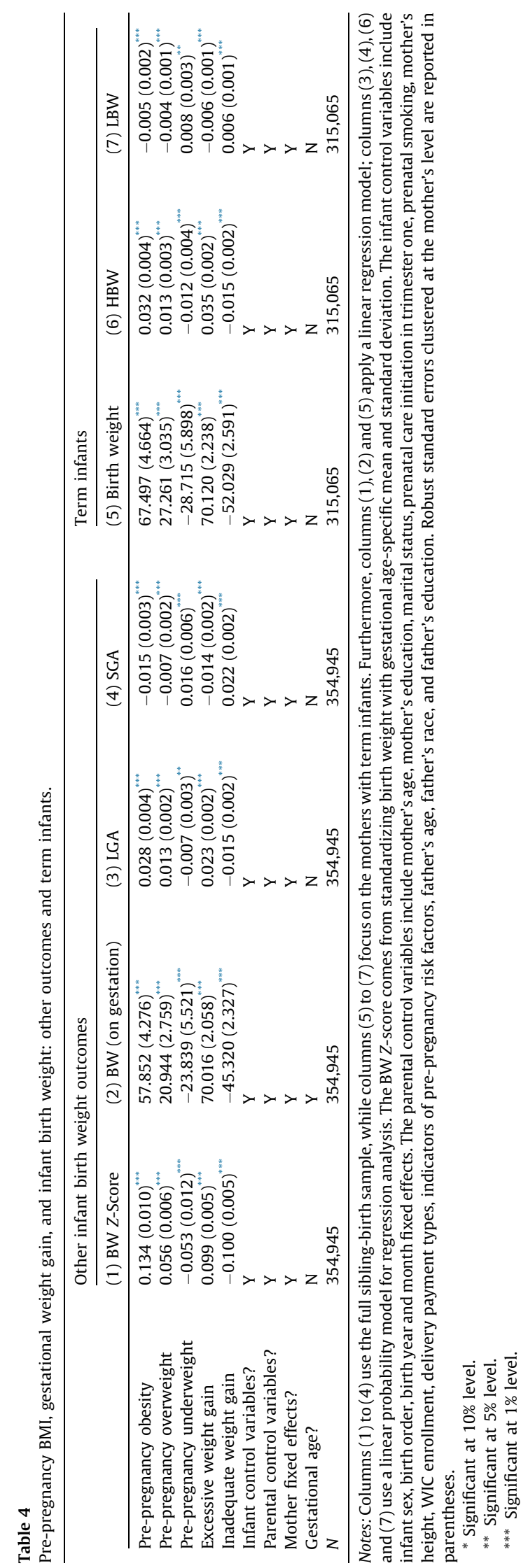


Table 5

Pre-pregnancy BMI, gestational weight gain, and infant birth weight: further robustness checks.

\begin{tabular}{|c|c|c|c|c|c|c|}
\hline & \multicolumn{3}{|c|}{ The first and second births } & \multicolumn{3}{|c|}{ Gestational weight gain $>0$} \\
\hline & (1) Birth weight & (2) HBW & (3) LBW & (4) Birth weight & (5) HBW & (6) LBW \\
\hline Pre-pregnancy obesity & $72.077(6.839)^{* * * *}$ & $0.028(0.005)^{* * *}$ & $-0.010(0.003)^{* * * *}$ & $72.709(5.674)^{* * *}$ & $0.029(0.004)^{* * *}$ & $-0.011(0.003)^{* * * *}$ \\
\hline Pre-pregnancy overweight & $21.660(4.362)^{* * * *}$ & $0.011(0.003)^{* * *}$ & $-0.005(0.002)^{* * *}$ & $25.828(3.559)^{* * * *}$ & $0.011(0.003)^{* * * *}$ & $-0.006(0.002)^{* * * *}$ \\
\hline Pre-pregnancy underweight & $-24.750(8.341)^{* * * *}$ & $-0.013(0.004)^{* * * *}$ & $0.012(0.005)^{*+4}$ & $-27.577(6.843)^{* * * *}$ & $-0.010(0.004)^{* * * *}$ & $0.011(0.004)^{* * * *}$ \\
\hline Excessive weight gain & $136.481(3.188)^{*+*}$ & $0.039(0.002)^{* 2 *}$ & $-0.025(0.002)^{* * *}$ & $128.082(2.554)^{* * * *}$ & $0.040(0.002)^{* * *}$ & $-0.026(0.001)^{* * *}$ \\
\hline Inadequate weight gain & $-63.120(3.977)^{*+* *}$ & $-0.010(0.002)^{* * *}$ & $0.022(0.002)^{* * * *}$ & $-70.260(3.283)^{* * * *}$ & $-0.014(0.002)^{* * *}$ & $0.021(0.002)^{*+*}$ \\
\hline Infant control variables? & $\mathrm{Y}$ & $\mathrm{Y}$ & $\mathrm{Y}$ & $\mathrm{Y}$ & $\mathrm{Y}$ & $\mathrm{Y}$ \\
\hline Parental control variables? & $\mathrm{Y}$ & $\mathrm{Y}$ & $\mathrm{Y}$ & $\mathrm{Y}$ & $\mathrm{Y}$ & $\mathrm{Y}$ \\
\hline Mother fixed effects? & Y & $\mathrm{Y}$ & $\mathrm{Y}$ & $\mathrm{Y}$ & $\mathrm{Y}$ & $\mathrm{Y}$ \\
\hline$N$ & 225,994 & 225,994 & 225,994 & 333,043 & 333,043 & 333,043 \\
\hline
\end{tabular}

Notes: Columns (1)-(3) focus on the mothers who had delivered only the first and second live birth in the sampling period. Columns (4)-(6) restrict the sample to the mothers with gestational weight gain greater than zero. Furthermore, columns (1) and (4) apply a linear regression model; columns (2), ( 3 ), (5) and (6) use a linear probability model for regression analysis. The infant control variables include infant sex, birth order, birth year and month fixed effects. The parental control variables include mother's age, mother's education, marital status, prenatal care initiation in trimester one, prenatal smoking, mother's height, WIC enrollment, delivery payment types, indicators of pre-pregnancy risk factors, father's age, father's race, and father's education. Robust standard errors clustered at the mother's level are reported in parentheses.

* Significant at $10 \%$ level.

** Significant at $5 \%$ level.

*** Significant at $1 \%$ level.

Table 5 performs two other robustness checks. First, while the samples used above have marked variations in the newborn birth order, columns (1)-(3) use a more homogenous sibling subsample which consists of only the first- and second-born infants. The new coefficient estimates of pre-pregnancy BMI and gestational weight gain are similar to their counterparts in Table 2. Second, gestational weight gain takes negative values for a small

Table 6

Pre-pregnancy BMI, gestational weight gain, and infant birth weight: heterogeneous effects and logit regression.

\begin{tabular}{|c|c|c|c|c|c|}
\hline & \multicolumn{3}{|c|}{ Heterogeneous effects of weight gain } & \multicolumn{2}{|c|}{ Logit (odds ratios) } \\
\hline & (1) Birth weight & (2) HBW & (3) LBW & (4) HBW & (5) LBW \\
\hline Pre-pregnancy obesity & $79.205(7.294)^{* * * *}$ & $0.024(0.005)^{* * *}$ & $-0.018(0.005)^{* * * *}$ & $1.477(0.077)^{* * *}$ & $0.633(0.050)^{* * *}$ \\
\hline Pre-pregnancy overweight & $39.883(5.208)^{* * * *}$ & $0.014(0.003)^{* * *}$ & $-0.017(0.003)^{* * * *}$ & $1.192(0.043)^{* * * *}$ & $0.785(0.042)^{* * *}$ \\
\hline Pre-pregnancy underweight & $-25.303(8.228)^{* * * *}$ & $-0.008(0.004)^{* * *}$ & $0.010(0.005)^{*}$ & $0.839(0.082)^{*}$ & $1.312(0.108)^{* * *}$ \\
\hline Excessive weight gain & & & & $1.702(0.048)^{* * *}$ & $0.466(0.017)^{* * *}$ \\
\hline Inadequate weight gain & & & & $0.811(0.031)^{* * *}$ & $1.406(0.054)^{* * *}$ \\
\hline $\begin{array}{l}\text { Excessive weight gain for } \\
\text { obese women }\end{array}$ & $105.119(5.944)^{* * *}$ & $0.039(0.004)^{* * *}$ & $-0.014(0.004)^{*+* *}$ & & \\
\hline $\begin{array}{l}\text { Excessive weight gain for } \\
\text { overweight women }\end{array}$ & $109.334(4.816)^{* * *}$ & $0.030(0.003)^{* * *}$ & $-0.017(0.003)^{* * *}$ & & \\
\hline $\begin{array}{l}\text { Excessive weight gain for } \\
\text { normal weight women }\end{array}$ & $127.474(3.169)^{* * *}$ & $0.032(0.002)^{* * *}$ & $-0.028(0.002)^{* * * *}$ & & \\
\hline $\begin{array}{l}\text { Excessive weight gain for } \\
\text { underweight women }\end{array}$ & $141.729(10.800)^{* * *}$ & $0.029(0.006)^{* * *}$ & $-0.027(0.007)^{* * *}$ & & \\
\hline $\begin{array}{l}\text { Inadequate weight gain for } \\
\text { obese women }\end{array}$ & $-44.081(10.379)^{* * * *}$ & $-0.008(0.005)^{*}$ & $0.024(0.003)^{* * *}$ & & \\
\hline $\begin{array}{l}\text { Inadequate weight gain for } \\
\text { overweight women }\end{array}$ & $-67.624(7.878)^{* * *}$ & $-0.015(0.004)^{* * *}$ & $0.029(0.004)^{* * *}$ & & \\
\hline $\begin{array}{l}\text { Inadequate weight gain for } \\
\text { normal weight women }\end{array}$ & $-61.510(3.738)^{* * *}$ & $-0.015(0.002)^{* * * *}$ & $0.020(0.004)^{* * *}$ & & \\
\hline $\begin{array}{l}\text { Inadequate weight gain for } \\
\text { underweight women }\end{array}$ & $-95.861(14.573)^{* * *}$ & $-0.014(0.005)^{* * *+}$ & $0.030(0.017)^{*}$ & & \\
\hline Infant control variables? & $\mathrm{Y}$ & $\mathrm{Y}$ & $\mathrm{Y}$ & $\mathrm{Y}$ & $\mathrm{Y}$ \\
\hline Parental control variables? & $\mathrm{Y}$ & $\mathrm{Y}$ & $\mathrm{Y}$ & $\mathrm{Y}$ & $\mathrm{Y}$ \\
\hline Mother fixed effects? & $\mathrm{Y}$ & $\mathrm{Y}$ & $\mathrm{Y}$ & $\mathrm{Y}$ & $\mathrm{Y}$ \\
\hline$N$ & 354,945 & 354,945 & 354,945 & 354,945 & 354,945 \\
\hline
\end{tabular}

Notes: All the regressions use the full sibling-birth sample which consists of all the mothers having delivered two or three live births in the state of Pennsylvania in 2003-2010 and in the state of Washington in 2003-2006. Furthermore, column (1) applies a linear regression model; columns (2) and (3) use a linear probability model; columns (4) and (5) apply a fixed effects logit model for regression analysis. The infant control variables include infant sex, birth order, birth year and month fixed effects. The parental control variables include mother's age, mother's education, marital status, prenatal care initiation in trimester one, prenatal smoking, mother's height, WIC enrollment, delivery payment types, indicators of pre-pregnancy risk factors, father's age, father's race, and father's education. Robust standard errors clustered at the mother's level are reported in parentheses.

* Significant at $10 \%$ level.

** Significant at $5 \%$ level.

*** Significant at $1 \%$ level. 
proportion of the mothers in the original sample. One explanation is that some pregnant women attempted weight loss (Bodnar et al., 2010). However, as another possibility, such negative weight changes are due to recall error which may systematically bias the estimates. Columns (4)-(6) address this concern by studying only the mothers with weight gain greater than zero. Still, this sample restriction does not significantly alter the results.

Several additional sensitivity checks are also conducted, with the full results available upon request. One, a new cutoff of $4500 \mathrm{~g}$ for high birth weight does not materially affect the results, when we compare the new estimates with those in Table 2 relative to respective sample means. Two, a series of Hausman specification tests all reject the null hypothesis that models with mother random effects are preferred to the baseline model with mother fixed effects. Three, no evidence is found that previous birth outcomes (e.g., birth weight) significantly affects a woman's future pre-pregnancy BMI or gestational weight gain. This finding corroborates the validity of the strict exogeneity condition, a necessary condition to get unbiased estimates in Eq. (1). Four, when a continuous gestational weight gain enters Eq. (1) and pre-pregnancy BMI is not controlled for, the regression results are similar to Ludwig and Currie (2010) which uses sibling births to study the birth weight effect of weight gain subject to lack of data on preconception BMI. Interestingly, after prepregnancy BMI is controlled to correct for this omitted variable bias in this study, we find the estimated effect of weight gain on birth weight becomes about 30\% larger.

Table 6 extends the benchmark analysis in two ways. It first explores if the effects of gestational weight gain differ by BMI category. Columns (1) and (3) show the effects of excessive and inadequate weight gain on newborn birth weight and LBW are stronger for underweight women. However, the impacts of unhealthy weight gain on HBW are generally similar across different pre-pregnancy BMI categories except for the obese women (column 2). The last two columns use the logit model with mother fixed effects in Eq. (2). On one hand, column (4) indicates the odds of having a HBW infant are 1.19 and 1.48 times greater for overweight and obese women, compared to normal weight women. The odds of delivering a HBW baby for women with excessive gestational weight gain is about 1.7 times larger than the odds for women with appropriate weight gain. On the other hand, compared with the normal weight women, the odds of giving birth to a LBW infant is 1.31 times greater for underweight women (column 5). In addition, the odds of having a LBW baby is 1.41 times larger for women with inadequate weight gain than that for women with appropriate weight gain. Finally, as to the effect direction, the main findings above (Tables 2, 4 and 6) are generally consistent with the previous studies such as Frederick et al. (2008) and Bodnar et al. (2010). However, estimates in the present study are more precise and smaller.

\section{Conclusion}

Poor birth outcomes such as HBW and LBW impose a huge burden on families, the healthcare care system, education, and social services. As such, improving the health and well-being of infants has been a crucial public health goal in the United States. Both pre-pregnancy BMI and gestational weight gain are two key determinants of infant health. However, the exact relationship between the two inputs and newborn birth weight has not been well understood. This article uses a large sample of sibling births to provide new evidence on this issue. The preliminary analysis indicates about $49 \%$ of the newborns' mothers were obese, overweight, or underweight before pregnancy and $71 \%$ of them had excessive or inadequate weight in pregnancy. The in-depth regression analysis controls for the mother fixed effects and a rich set of infant, mother, and family characteristics. Overall, the benchmark results show preconception overweight, preconception obesity, and excessive weight gain during pregnancy increase the risk of having a HBW baby by $1.3,3$, and 3.9 percentage points, respectively. In contrast, underweight before pregnancy and inadequate gestational weight gain result in an increase in the LBW incidence by 1.4 and 2 percentage points.

The baseline results are robust in a variety of sensitivity checks such as using an alternative weight gain guideline, stratifying the sample by number of sibling births or by state, applying other birth weight outcomes conditional on gestational age, restricting the sample to the women with positive gestational weight changes, and exploring heterogeneous effects of weight gain by BMI category. This study has the following limitations. First, it only looks at infant health at birth but does not examine any outcome later in life or maternal health during childbirth and the postpartum period. Second, there can be reporting errors on maternal height and weight in birth certificates, in addition to missing information for some fathers. Furthermore, the dataset neither codes preterm birth by type nor the timing of weight gain through pregnancy ${ }^{15}$. Third, the present analysis is limited to two states. Addressing these issues opens up avenues for future research.

The high incidence of adverse birth outcomes provides large scope for early intervention during the preconception and antenatal periods (Doyle et al., 2009). The findings of this study suggest one approach to enhance infant health is promoting healthy weight before pregnancy. To achieve this goal, it is important to offer women of reproductive age guidelines and programs on diet, nutrition, physical activity, and weight management. The second strategy is to prevent inappropriate weight gain among pregnant women. Integration of the two approaches in practice is particularly encouraging. The partnership of the Centers for Disease Control and Prevention, the Association of Maternal and Child Health Programs and CityMatCH is making such an effort, by building federal, state, and local capacity to promote healthy weight among women before pregnancy and prevent inappropriate weight gain during pregnancy.

\footnotetext{
15 Some studies report nonlinear weight gain through different stages in pregnancy (Hediger et al., 1989; Abrams and Selvin, 1995; Hickey et al., 1996; Saad et al., 2012). However, the dataset of this study does not code maternal weight by trimester.
} 


\section{Acknowledgements}

This paper benefits from helpful comments by Josh Berning, Jeremy Bray, Karen Conway, Anca Cotet, Angela Dills, Zheng Fang, Gregory Gilpin, Jennifer Graves, Tiffany Green, Robert Pollak, Kathleen M. Rasmussen, Anna Maria Siega-Riz and participants of Association for Public Policy Analysis and Management Research Conference, Eastern Economic Association Meeting, Southern Economic Association Meeting, and the Work, Families and Public Policy Workshop in Washington University in Saint Louis. Research grants are provided by the Center for New Institutional Social Sciences at Washington University in Saint Louis and Walker College of Business Dean's Club at Appalachian State University.

\section{Appendix A}

Table A.1

Institute of medicine, 2009 and 1990 guidelines of gestational weight gain for women with singleton fetuses: by pre-pregnancy BMI category.

\begin{tabular}{|c|c|c|}
\hline \multirow[t]{2}{*}{ BMI category $\left(\mathrm{kg} / \mathrm{m}^{2}\right)$} & \multicolumn{2}{|l|}{2009 guideline } \\
\hline & $\begin{array}{l}\text { (1) Recommended } \\
\text { total weight } \\
\text { gain }(\mathrm{kg})\end{array}$ & $\begin{array}{l}\text { (2) Rates of } \\
\text { weight gain } \\
\text { in } 2 \text { nd and } \\
\text { 3rd trimester } \\
\text { in kg/week: } \\
\text { mean and } \\
\text { range (in the } \\
\text { parentheses) }\end{array}$ \\
\hline Underweight (<18.5) & $12.5-18$ & $0.51(0.44-0.58)$ \\
\hline Normal weight (18.5-25) & $11.5-16$ & $0.42(0.35-0.50)$ \\
\hline Overweight (25-30) & $7-11.5$ & $0.28(0.23-0.33)$ \\
\hline \multirow[t]{3}{*}{ Obese $(\geq 30)$} & $5-9$ & $0.22(0.17-0.27)$ \\
\hline & \multicolumn{2}{|l|}{1990 Guideline } \\
\hline & $\begin{array}{l}\text { Recommended } \\
\text { total weight } \\
\text { gain }(\mathrm{kg})\end{array}$ & $\begin{array}{l}\text { Rates of weight } \\
\text { gain in } 2 \text { nd and } \\
\text { 3rd trimester in } \\
\text { kg/week: mean }\end{array}$ \\
\hline Underweight (<19.8) & $12.5-18$ & 0.5 \\
\hline Normal weight (19.8-26) & $11.5-16$ & 0.4 \\
\hline Overweight (26-29) & $7-11.5$ & 0.3 \\
\hline Obese ( $\geq 29)$ & $>6.8$ & Not specified \\
\hline
\end{tabular}

Source: IOM (1990), IOM (2009), Rasmussen et al. (2009).

\section{References}

Abenhaim, H.A., Kinch, R.A., Morin, L., Benjamin, A., Usher, R., 2007. Effect of pre-pregnancy body mass index categories on obstetrical and neonatal outcomes. Archives of Gynecology and Obstetrics 275 (1), 39-43.

Abrams, B., Altman, S.L., Pickett, K.E., 2000. Pregnancy weight gain: still controversial. The American Journal of Clinical Nutrition 71 (5), 12331241.

Abrams, B., Selvin, S., 1995. Maternal weight gain pattern and birth weight. Obstetrics \& Gynecology 86 (2), 163-169.

Almond, D., Chay, K.Y., Lee, D.S., 2005. The costs of low birth weight. The Quarterly Journal of Economics 120 (3), 1031-1083.

Almond, D., Currie, J., 2011. Human capital development before age five. Handbook of Labor Economics, vol. 4. The North-Holland Press, Amsterdam, Netherlands, pp. 1315-1486.

Barker, D.J., 1990. The fetal and infant origins of adult disease. British Medical Journal 301 (6761), 1111.

Behrman, J.R., Rosenzweig, M.R., 2004. Returns to birthweight. Review of Economics and Statistics 86 (2), 586-601.
Black, S.E., Devereux, P.J., Salvanes, K.G., 2007. From the cradle to the labor market? The effect of birth weight on adult outcomes. The Quarterly Journal of Economics 122 (1), 409-439.

Bodnar, L.M., Siega-Riz, A.M., Simhan, H.N., Himes, K.P., Abrams, B., 2010. Severe obesity, gestational weight gain, and adverse birth outcomes. The American Journal of Clinical Nutrition 91 (6), 1642-1648.

Carreno, C.A., Clifton, R.G., Hauth, J.C., Myatt, L., Roberts, J.M., Spong, C.Y., Varner, M.W., Thorp, J.M., Mercer, B.M., Peaceman, A.M., Ramin, S.M., Carpenter, M.W., Sciscione, A., Tolosa, J.E., Sorokin, Y., 2012. Excessive early gestational weight gain and risk of gestational diabetes mellitus in nulliparous women. Obstetrics and Gynecology 119 (6), 1227-1233.

Cesur, R., Kelly, I.R., 2010. From cradle to classroom: high birth weight and cognitive outcomes. Forum for Health Economics \& Policy 13 (2), 1-26.

Conley, D., Bennett, N.G., 2000. Is biology destiny? Birth weight and life chances. American Sociological Review 65, 458-467.

Crane, J.M., White, J., Murphy, P., Burrage, L., Hutchens, D., 2009. The effect of gestational weight gain by body mass index on maternal and neonatal outcomes. Journal of Obstetrics and Gynecology Canada 31 (1), 28-35.

Currie, J., Hyson, R., 1999. Is the impact of health shocks cushioned by socioeconomic status? The case of low birthweight. American Economic Review Papers and Proceedings 89 (2), 245-250.

Danielzik, S., Czerwinski-Mast, M., Langnäse, K., Dilba, B., Müller, M.J., 2004. Parental overweight, socioeconomic status and high birth weight are the major determinants of overweight and obesity in 5-7 year-old children: baseline data of the Kiel Obesity Prevention Study (KOPS). International Journal of Obesity 28 (11), 1494-1502.

DeVader, S.R., Neeley, H.L., Myles, T.D., Leet, T.L., 2007. Evaluation of gestational weight gain guidelines for women with normal pre-pregnancy body mass index. Obstetrics \& Gynecology 110 (4), 745-751.

Dietz, P.M., Bombard, J.M., Hutchings, Y.L., Gauthier, J.P., Gambatese, M.A., Ko, J.Y., Martin, J.A., Callaghan, W.M., 2014. Validation of obstetric estimate of gestational age on US birth certificates. American Journal of Obstetrics and Gynecology 210 (4), 335-337.

Doherty, D.A., Magann, E.F., Francis, J., Morrison, J.C., Newnham, J.P., 2006. Pre-pregnancy body mass index and pregnancy outcomes. International Journal of Gynecology \& Obstetrics 95 (3), 242-247.

Doyle, O., Harmon, C.P., Heckman, J.J., Tremblay, R.E., 2009. Investing in early human development: timing and economic efficiency. Economics \& Human Biology 7 (1), 1-6.

Fisher, S.C., Kim, S.Y., Sharma, A.J., Rochat, R., Morrow, B., 2013. Is obesity still increasing among pregnant women? Pre-pregnancy obesity trends in 20 states, 2003-2009. Preventive Medicine 56 (6), 372-378.

Frederick, I.O., Williams, M.A., Sales, A.E., Martin, D.P., Killien, M., 2008. Pre-pregnancy body mass index, gestational weight gain, and other maternal characteristics in relation to infant birth weight. Maternal and Child Health Journal 12 (5), 557-567.

Gilboa, S.M., Correa, A., Alverson, C.J., 2008. Use of spline regression in an analysis of maternal pre-pregnancy body mass index and adverse birth outcomes: does it tell us more than we already know? Annals of Epidemiology 18 (3), 196-205.

Hack, M., Klein, N.K., Taylor, H.G., 1995. Long-term developmental outcomes of low birth weight infants. The Future of Children 5 (1), 176196.

Harder, T., Rodekamp, E., Schellong, K., Dudenhausen, J.W., Plagemann, A., 2007. Birth weight and subsequent risk of type 2 diabetes: a metaanalysis. American Journal of Epidemiology 165 (8), 849-857.

Hediger, M.L., Scholl, T.O., Belsky, D.H., Ances, I.G., Salmon, R.W., 1989. Patterns of weight gain in adolescent pregnancy: effects on birth weight and preterm delivery. Obstetrics \& Gynecology 74 (1), 6-12.

Hickey, C.A., Cliver, S.P., McNeal, S.F., Hoffman, H.J., Goldenberg, R.L., 1996. Prenatal weight gain patterns and birth weight among nonobese black and white women. Obstetrics \& Gynecology 88, 490-496.

Hjalgrim, L.L., Westergaard, T., Rostgaard, K., Schmiegelow, K., Melbye, M., Hjalgrim, H., Engels, E.A., 2003. Birth weight as a risk factor for childhood leukemia: a meta-analysis of 18 epidemiologic studies. American Journal of Epidemiology 158 (8), 724-735.

Institute of Medicine, 1990. Nutrition during Pregnancy: Part I, Weight Gain; Part II, Nutrient Supplements. National Academy Press, Washington, DC.

Institute of Medicine, 2009. Weight Gain during Pregnancy: Reexamining the Guidelines. National Academy Press, Washington, DC.

Jansson, N., Nilsfelt, A., Gellerstedt, M., Wennergren, M., RossanderHulthén, L., Powell, T.L., Jansson, T., 2008. Maternal hormones linking maternal body mass index and dietary intake to birth weight. American Journal of Clinical Nutrition 87 (6), 1743-1749.

Kim, S.Y., Dietz, P.M., England, L., Morrow, B., Callaghan, W.M., 2007. Trends in pre-pregnancy obesity in nine states, 1993-2003. Obesity 15 (4), 986-993.

Kirkegaard, I., Obel, C., Hedegaard, M., Henriksen, T.B., 2006. Gestational age and birth weight in relation to school performance of 10 -year-old 
children: a follow-up study of children born after 32 completed weeks. Pediatrics 118 (4), 1600-1606.

Ludwig, D.S., Currie, J., 2010. The association between pregnancy weight gain and birthweight: a within-family comparison. The Lancet 376 , 984-990.

Mandl, L.A., Costenbader, K.H., Simard, J.F., Karlson, E.W., 2009. Is birthweight associated with risk of rheumatoid arthritis? Data from a large cohort study. Annals of the Rheumatic Diseases 68 (4), 514-518.

McDonald, S.D., Han, Z., Mulla, S., Beyene, J., 2010. Overweight and obesity in mothers and risk of preterm birth and low birth weight infants: systematic review and meta-analyses. British Medical Journal 341, $1-20$.

McDonald, S.D., Han, Z., Mulla, S., Lutsiv, O., Lee, T., Beyene, J., 2011. High gestational weight gain and the risk of preterm birth and low birth weight: a systematic review and meta-analysis. Journal of Obstetrics and Gynecology Canada 33 (12), 1223-1233.

National Center for Health Statistics, 2014. Health, United States, 2013 With Special Feature on Prescription Drugs. National Center for Health Statistics, Hyattsville, MD.

Rasmussen, K.M., Abrams, B., Bodnar, L.M., Butte, N.F., Catalano, P.M. Siega-Riz, A.M., 2010. Recommendations for weight gain during pregnancy in the context of the obesity epidemic. Obstetrics \& Gynecology 116 (5), 1191-1195.

Rasmussen, K.M., Catalano, P.M., Yaktine, A.L., 2009. New guidelines for weight gain during pregnancy: what obstetrician/gynecologists should know. Current Opinion in Obstetrics and Gynecology 21 521-526.

Richards, M., Hardy, R., Kuh, D., Wadsworth, M.E., 2001. Birth weight and cognitive function in the British 1946 birth cohort: longitudinal population based study. BMJ: British Medical Journal $322,199-204$
Rode, L., Hegaard, H.K., Kjargaard, H., Moller, L.F., Tabor, A., Ottesen, B. 2007. Association between maternal weight gain and birth weight. Obstetrics \& Gynecology 109 (6), 1309-1315.

Saad, K., Shahar, D.R., Fraser, D., Vardi, H., Friger, M., Bolotin, A., Freedman, L.S., 2012. Adequacy of usual dietary intake and nutritional status among pregnant women in the context of nutrition transition: the DEPOSIT Study. British Journal of Nutrition 108 (10), 1874-1883.

Siega-Riz, A.M., Deierlein, A., Stuebe, A., 2010. Implementation of the new institute of medicine gestational weight gain guidelines. Journal of Midwifery \& Women's Health 55 (6), 512-519.

Siega-Riz, A.M., Laraia, B., 2006. The implications of maternal overweight and obesity on the course of pregnancy and birth outcomes. Maternal and Child Health Journal 10 (1), 153-156.

Thorsdottir, I., Torfadottir, J.E., Birgisdottir, B.E., Geirsson, R.T., 2002. Weight gain in women of normal weight before pregnancy: complications in pregnancy or delivery and birth outcome. Obstetrics \& Gynecology 99 (5), 799-806.

Voigt, M., Heineck, G., Hesse, V., 2004. The relationship between maternal characteristics, birth weight and pre-term delivery: evidence from Germany at the end of the 20th century. Economics \& Human Biology 2 (2), 265-280.

Wei, J.N., Li, H.Y., Sung, F.C., Lin, C.C., Chiang, C.C., Li, C.Y., Chuang, L.M. 2007. Birth weight correlates differently with cardiovascular risk factors in youth. Obesity 15 (6), 1609-1616.

Wier, M.L., Pearl, M., Kharrazi, M., 2007. Gestational age estimation on United States livebirth certificates: a historical overview. Paediatric and Perinatal Epidemiology 21 (s2), 4-12.

Wooldridge, J.M., 2002. Econometric Analysis of Cross Section and Panel Data. MIT Press, Cambridge, MA.

World Health Organization, 1995. Physical Status: the Use and Interpretation of Anthropometry. WHO Technical Report Series 854, WHO Press, Switzerland. 